\title{
Facial-Expression Affective Attributes and their Configural Correlates: Components and Categories
}

\author{
David L. Bimler ${ }^{1}$ and Galina V. Paramei ${ }^{2}$ \\ ${ }^{1}$ Massey University (New Zealand) $\quad{ }^{2}$ Darmstadt University of Technology
}

\begin{abstract}
The present study investigates the perception of facial expressions of emotion, and explores the relation between the configural properties of expressions and their subjective attribution. Stimuli were a male and a female series of morphed facial expressions, interpolated between prototypes of seven emotions (happiness, sadness, fear, anger, surprise and disgust, and neutral) from Ekman and Friesen (1976). Topographical properties of the stimuli were quantified using the Facial Expression Measurement (FACEM) scheme. Perceived dissimilarities between the emotional expressions were elicited using a sorting procedure and processed with multidimensional scaling. Four dimensions were retained in the reconstructed facial-expression space, with positive and negative expressions opposed along D1, while the other three dimensions were interpreted as affective attributes distinguishing clusters of expressions categorized as "Surprise-Fear," "Anger," and "Disgust." Significant relationships were found between these affective attributes and objective facial measures of the stimuli. The findings support a componential explanatory scheme for expression processing, wherein each component of a facial stimulus conveys an affective value separable from its context, rather than a categorical-gestalt scheme. The findings further suggest that configural information is closely involved in the decoding of affective attributes of facial expressions. Configural measures are also suggested as a common ground for dimensional as well as categorical perception of emotional faces.
\end{abstract}

Keywords: facial expression, emotion, facial configuration, affective dimensions

\begin{abstract}
Este estudio investiga la percepción de las expresiones faciales de la emoción y explora la relación entre las propiedades configurales de las expresiones y su atribución subjetiva. Los estímulos eran una serie de expresiones faciales transformadas por ordenador, interpuestas entre los prototipos de siete emociones (felicidad, tristeza, miedo, ira, sorpresa, asco y neutral) tomados de Ekman y Friesen (1976). Las propiedades topográficas de los estímulos se cuantificaron mediante el esquema Facial Expression Measurement (FACEM). Las disimilaridades percibidas entre las expresiones emocionales se elicitaron mediante un procedimiento de clasificación y se procesaron con escalonamiento multidimensional. Se retuvieron cuatro dimensiones en el espacio facial-expresión reconstruido, con expresiones positivas y negativas contrapuestas a lo largo de D1, y las restantes tres dimensiones se interpretaron como atributos afectivos, distinguiendo clusters de expresiones clasificadas como "Sorpresa/Miedo", "Ira", y "Asco". Se hallaron relaciones significativas entre estos atributos afectivos y las medidas faciales objetivas de los estímulos. Los resultados apoyan un esquema explicativo componencial para el procesamiento de las expresiones, en el que cada componente de un estímulo facial conlleva un valor afectivo separable de su contexto, más que un esquema categórico de tipo Gestalt. Además sugieren que la información configural juega un papel importante en la decodificación de los atributos afectivos de las expresiones faciales Además, sugieren que las medidas configurales constituyen en terreno común de la percepción dimensional y categórica de las caras emocionales.
\end{abstract}

Palabras clave: expresión facial, emoción, configuración facial, dimensiones afectivas

We are grateful to Paul Ekman for permission to use facial-expression photographs and for the FACS analyses described in the text. We thank Mary Katsikitis and anonymous reviewers for critical and helpful comments of earlier versions of the article. The sorting data were made available by John Kirkland of Massey University (NZ). R. Surville and S. Lane performed the arduous task of morphing the stimuli. Assistance of Ute Lobisch in preparation of figures is gratefully acknowledged. GVP was supported by the Humboldt Foundation (Germany) and Eastern European collaborative grant JSMF/95-59 from the McDonnell Foundation (Oxford, UK).

Correspondence concerning this article should be addressed to David L. Bimler, Department of Health and Human Development, Massey University, Private Bag 11-222, Palmerston North, New Zealand. E-mail: d.bimler@massey.ac.nz 
There is general acceptance that facial expressions (FEs) convey information about one's emotional state (e.g., Ekman, 1993; Russell \& Fernández-Dols, 1997). This implies that the processes of encoding and decoding of emotions are two sides of a coin, so that perception of emotional expressions can only be fully understood when examined in conjunction with how the information is encoded in a facial display.

An expression can be described at a number of levels: as an aggregate of individual features such as raised eyebrows and opened mouth; as a Gestalt emerging from its features; as a configuration (i.e., topographical relations between facial landmarks). It is an open question which level of description is best targeted by research into the facial communication of affect.

The present paper considers three models at different levels for the decoding of facial displays, and searches for evidence that might discriminate between them. We focus on the role played in the perception of FEs by the attribution of continuously-varying affective meanings. This approach calls for continuous judgments, in which observers rate expression stimuli along researcher-nominated scales, or such scales and ratings are inferred by analyzing the subjective dissimilarities among the stimuli with multidimensional scaling (MDS). This contrasts with the identification procedure, in which observers are presented with single photographs (or line drawings) depicting various emotions and are required to assign each expression to a category from a predetermined set.

The stimuli used here are a set of extensively studied FE photographs (Ekman \& Friesen, 1978) that reliably convey six basic emotion categories and a neutral state, plus arrays of photographic-quality morphed facial expressions derived from them. A subset of the stimuli has been previously mapped using MDS (Bimler \& Kirkland, 2001). Here, those results are extended to a broader gamut of blended expressions. Morphed emotional faces have received some attention within the dimensional paradigm (Takehara \& Suzuki, 2001). However, their main use has been in studies on categorical perception (Calder, Young, Perrett, Etcoff, \& Rowland, 1996; De Gelder, Teunisse, \& Benson, 1997; Hsu \& Young, 2004; Young et al., 1997).

\section{Three Explanatory Schemes for Decoding Facial Expressions}

Increasingly sophisticated systems have been developed for decomposing FEs into components (e.g., features and configural codes). Notably, the Facial Action Coding System or FACS (Ekman \& Friesen, 1978) itemizes underlying facial muscle activity in terms of Action Units (AUs). As yet, no consensus has emerged as to how such components work together. A single facial component is seldom confined to a single emotion label, but may appear in a range of expressions which crosses emotion-label boundaries (for a review, see Smith \& Scott, 1997). Conversely, the expressions grouped together under a given emotion label can be diverse, exhibiting AUs in a range of combinations, without necessarily sharing a single unit in common as the essential, defining feature of that emotional category (Alvarado, 1996; Wallbott \& RicciBitti, 1993).

Smith and Scott (1997) distinguished three explanatory schemes for facial-expression processing: pure componential, componential, and pure categorical models (or classes of models). Each model in this spectrum suggests an analogous interpretation of the structure of emotions themselves. The models also differ in their implications for the mechanisms of FE perception.

The pure componential model is the extreme position that a FE's affective meaning is simply the sum of contributions from its constituent features. Each feature qua component has an intrinsic affective value: This could be regarded as a vector in an affective-meaning space, in which a FE's location is the sum of its component's vectors. The dimensionality required for the space is an empirical question that invites scrutiny.

The componential model agrees that affective meanings can be validly ascribed to individual components, but also allows for a contribution from the face as a gestalt, so that the whole is more than the sum of its parts.

At the other extreme is the pure categorical model, in which a given component has no constant or intrinsic meaning, as affective value varies with context, and can only be ascribed to the facial composite-gestalt. Components do not work together in an additive way. This model predicts that the presence of a component in a range of combinations does not provide those combinations with a common element or trend traceable to the component. As the name "pure categorical" suggests, emotional decoding in this model relies upon categorical perception of FEs. One is led to postulate perceptual "modules" tuned to the facial composites displaying each basic emotion: where 'composite' is specified as a pattern of AUs (Calder, Young, Keane, \& Dean, 2000b; Etcoff \& Magee, 1992).

In the present study we parameterize the stimuli with quantitative measures, for comparison against their spatial coordinates in a MDS solution (derived from inter-stimulus judgments). Because the parameters are not independent, a high correlation between the locations of stimuli in a "FE space" and their values on a given parameter does not guarantee that the information afforded by that parameter was used by observers when making the judgments. However, it is compatible with such use; in particular, with componential use (i.e., as information about emotional attributes, continuous across categories). A number of strong correlations would support a central role in perception for components and their combinations. 


\section{Configural Parameters of the Affective Face}

Parameters at various levels of detail are possible, ranging from the molar level of a "linear / curved" distinction (Yamada, 1993) to a fine-grained, feature-by-feature description. An intermediate level of abstraction is provided by the topographical layout of the face-its configurationin the form of relationships among facial "landmarks." If the emotional content of FEs are viewed as signals of the expresser's emotional state (rather than as inadvertent leaks of information), the appropriate level to look for elemental components is at observable transformations of the facial integument, rather than the inferred, underlying mechanisms of muscle contraction. Convergent evidence for the role of "configuration," specified as a geometric description, can be adduced from neurophysiological (Young \& Yamane, 1992) and positron emission tomography (Sergent, Ohta, MacDonald, \& Zuck, 1994) studies on face recognition, which indicate that the neuronal representations of facial stimuli reflect the topographical arrangement of particular facial features. Because a given surface landmark is tugged in different directions by facial muscles that may be working in concert or in opposition, the fit between measurements of surface topography and AUs is not one-to-one.

The terms "configuration" and "configural" appear in the facial-expression literature with more than one sense. Sometimes they imply "holistic", "gestalt" or "global" information, distributed across the face in a non-local way so that the meaning of measurements depends on their context. To avoid ambiguity, we stress that we do not intend that sense here.

Since measuring distances is an easily-computerized way of reducing static displays of facial affect to objective parameters, considerable research has been inspired by the potential applications in facilitating communication between computer software and its users (Bartlett et al., 1996; Kearney \& McKenzie, 1993; Morishima, 1996). The perspective extends into the psychological domain where it is realized by the Facial Expression Measurement or FACEM system (Pilowsky, Thornton, \& Stokes, 1985). The FACEM measures are grounded on an anatomical model of muscle contraction, and require fewer parameters than the interface-related systems. They have been used in numerous studies and found to be efficient in quantitative discrimination of FEs in a range of general- and clinical-psychology studies (e.g., Katsikitis, Pridmore, \& Marzullo, 1999).

Previous studies found the FACEM measures to discriminate the prototype FACS expressions reasonably well (Benson \& Katsikitis, 1995; Pilowsky \& Katsikitis, 1994), that is, they can reproduce an a priori categorical structure. Such findings presuppose a categorical model rather than test it. A preliminary goal of the present study is to clarify whether the configural aspect of FEs is sufficient to account for the ability of observers to discriminate the continuum of facial emotions.
The primary objective, as described above, is to relate the FACEM descriptions of FEs, in configural (topographical) terms, to the dimensions of affective attribution.

\section{Geometrical Models for Representing the Affective Attributes of FEs}

Multidimensional scaling (MDS) constructs a spatial model in which each stimulus is represented by a point. The points are specified by coordinates, that is, their values on dimensions that represent underlying affective attributes, and are located so that the distances between them reproduce the dissimilarities perceived between the corresponding stimuli. MDS leaves room for the researcher to choose the number and nature of the dimensions extracted. Indeed, previous studies of facial emotion have disagreed about the number and the interpretation of the dimensions they found. Causes of these discrepancies include the range of stimuli sampled, the mode of presentation (photographs versus line drawings), and the different methodologies employed (reviewed in Smith \& Ellsworth, 1985), with the diversity of dimensions exacerbated by the solutions' rotational indeterminacy.

As the design of the present study belongs within the dimensional paradigm, we briefly review findings in this framework. Three dimensions are commonly found (Bimler \& Kirkland, 1997; Frijda, 1969; Lemay, Kirouack, \& Lacouture, 1995; Osgood, 1966; Paramei, 1996; Royal \& Hays, 1959). Since Schlosberg (1954), there has been consensus about one major dimension-variously described as "Valence," "Positive / Negative," or "Hedonic Tone"separating expressions of happiness from those of sadness, anger, etc. In two-dimensional solutions, the second axis is often found to range from neutral on one extreme and fear/surprise at the other; identified as "Activation" or "Arousal," it subsumes Schlosberg's "Attention / Rejection" and "Sleep / Tension" dimensions and reflects the overall intensity of the facial expression (Abelson \& Sermat, 1962; Alvarado, 1996; Bimler \& Kirkland, 1997; Cliff \& Young, 1968; Russell, 1980; Takehara \& Suzuki, 2001). However, a "control," or "personal agency" dimension also consistently appears, discriminating anger/disgust from fear/surprise (Alvarado \& Jameson, 1996; Frijda, 1969; Katsikitis, 1997; Nummenmaa, 1992); it shares many of the facial actions with the Attention dimension but is not identical with it (Smith \& Scott, 1997). Smith and Scott (Table 10.2) summarize the reported dimensions, and the facial components associated with each.

An unsatisfactory aspect of two-dimensional representations is that they predict the existence of "metameric" facial expressions which can be produced by more than one combination of prototype expressions, but these are not observed in practice (Morishima, 1996; Young et al., 1997). But although a two-dimensional solution may 
suppress salient ways of distinguishing facial expressions, a robust investigation of additional dimensions requires more items and data.

With 54 stimulus-points, the present solution sustains a more solid interpretation than the smaller numbers used in most studies, so it is discussed below at some length. In particular, we bring it to bear on issues of the dimensionality of "FE space," using the FACEM measures as an aid to its interpretation and optimal alignment.

We also consider a non-spatial tree structure or "dendrogram" as a complementary way of presenting perceived dissimilarities. Again, points are arranged so that the distances between them reproduce inter-stimulus dissimilarities as closely as possible, but here they consist of "leaf nodes" at the ends of a hierarchical structure of branches, and the distances between them are defined as the total length of branches traversed in moving from one node to another.

\section{Method}

\section{Stimuli}

From Pictures of Facial Affect (Ekman \& Friesen, 1976), seven photographs were selected (e57, e58, e60, e61, e63, e64, and e65), in which a female poser identified as MO expresses six basic emotions (Happiness, Surprise, Anger, Sadness, Fear, Disgust) and a Neutral state. The items are similar in terms of lighting, angle, etc., leaving the expressions themselves (in the form of displacements of easily-located facial landmarks) as the only way they differ. Monochrome slide images were scanned, and pairs of these digitized "parent" stimuli were blended in various proportions to synthesize another 47 expressions. Image interpolation was performed with morphing software (Design Studio) by identifying matching points in each "parent" pair. These points form the corners of topologically-equivalent triangular grids, and morphing consists of smoothly interpolating the shape and internal shading of each triangle. Each printed image measured $5 \times 8 \mathrm{~cm}$.

Along each continuum, morphs were spaced at equal intervals, so that (for instance) the four morphs between Sad and Surprise have the descriptive codes SaSu20 (consisting of 80\% Sad and 20\% Surprise), SaSu40, SaSu60, SaSu80 (Figure 1a). Some pairs of parents (e.g., Fear and Surprise) are relatively easily confused, so fewer morphs were interpolated between them. Note that it is not essential for the number of morphs on each continuum to match parental dissimilarity. These numbers and descriptive codes for the morphs are shown in Table 1.

Table 1

Codes for 7 Prototype Expressions and 47 Morphs, Showing Number of Morphs in each Sequence (Above Diagonal) and Descriptive Codes for each Morph (Below Diagonal)

\begin{tabular}{|c|c|c|c|c|c|c|c|}
\hline & $\begin{array}{l}\text { Anger } \\
\text { (A) }\end{array}$ & $\begin{array}{l}\text { Sadness } \\
\text { (Sa) }\end{array}$ & $\begin{array}{l}\text { Surprise } \\
(\mathrm{Su})\end{array}$ & $\begin{array}{l}\text { Happiness } \\
\text { (Ha) }\end{array}$ & $\begin{array}{c}\text { Fear } \\
(\mathrm{F})\end{array}$ & $\begin{array}{l}\text { Disgust } \\
\text { (D) }\end{array}$ & $\begin{array}{l}\text { Neutral } \\
(\mathrm{N})\end{array}$ \\
\hline A & - & 2 & 1 & 3 & - & - & 4 \\
\hline $\mathrm{Sa}$ & $\begin{array}{l}\text { ASa33, } \\
\text { ASa66 }\end{array}$ & - & 4 & 4 & 3 & 1 & 3 \\
\hline $\mathrm{Su}$ & ASu50 & $\begin{array}{c}\text { SaSu20, } \\
\text { SaSu40 } \\
\text { SaSu60, } \\
\text { SaSu80 }\end{array}$ & - & 1 & 1 & 2 & 3 \\
\hline $\mathrm{Ha}$ & $\begin{array}{c}\text { AH25, } \\
\text { AH50, AH75 }\end{array}$ & $\begin{array}{l}\mathrm{SaH} 20, \mathrm{SaH} 40 \\
\mathrm{SaH} 60, \mathrm{SaH} 80\end{array}$ & SuH50 & - & 3 & 3 & 2 \\
\hline $\mathrm{F}$ & - & $\begin{array}{c}\text { FSa25, FSa50 } \\
\text { FSa75 }\end{array}$ & FSu50 & $\begin{array}{c}\text { FH25, } \\
\text { FH50 FH75 }\end{array}$ & - & 1 & 4 \\
\hline D & - & DSa50 & $\begin{array}{l}\text { DSu33, } \\
\text { DSu66 }\end{array}$ & $\begin{array}{l}\text { DH25, } \\
\text { DH50 } \\
\text { DH75 }\end{array}$ & DF50 & - & 3 \\
\hline $\mathrm{N}$ & $\begin{array}{l}\text { AN20, AN40 } \\
\text { AN60, AN80 }\end{array}$ & $\begin{array}{c}\text { SaN25, SaN50 } \\
\text { SaN75 }\end{array}$ & $\begin{array}{l}\text { SuN25, } \\
\text { SuN50 } \\
\text { SuN75 }\end{array}$ & HN50 & $\begin{array}{l}\text { FN20, } \\
\text { FN40 } \\
\text { FN60, } \\
\text { FN80 }\end{array}$ & $\begin{array}{l}\text { DN25, } \\
\text { DN50 } \\
\text { DN75 }\end{array}$ & - \\
\hline
\end{tabular}

Note. The number refers to $\%$ of the second descriptive code. 

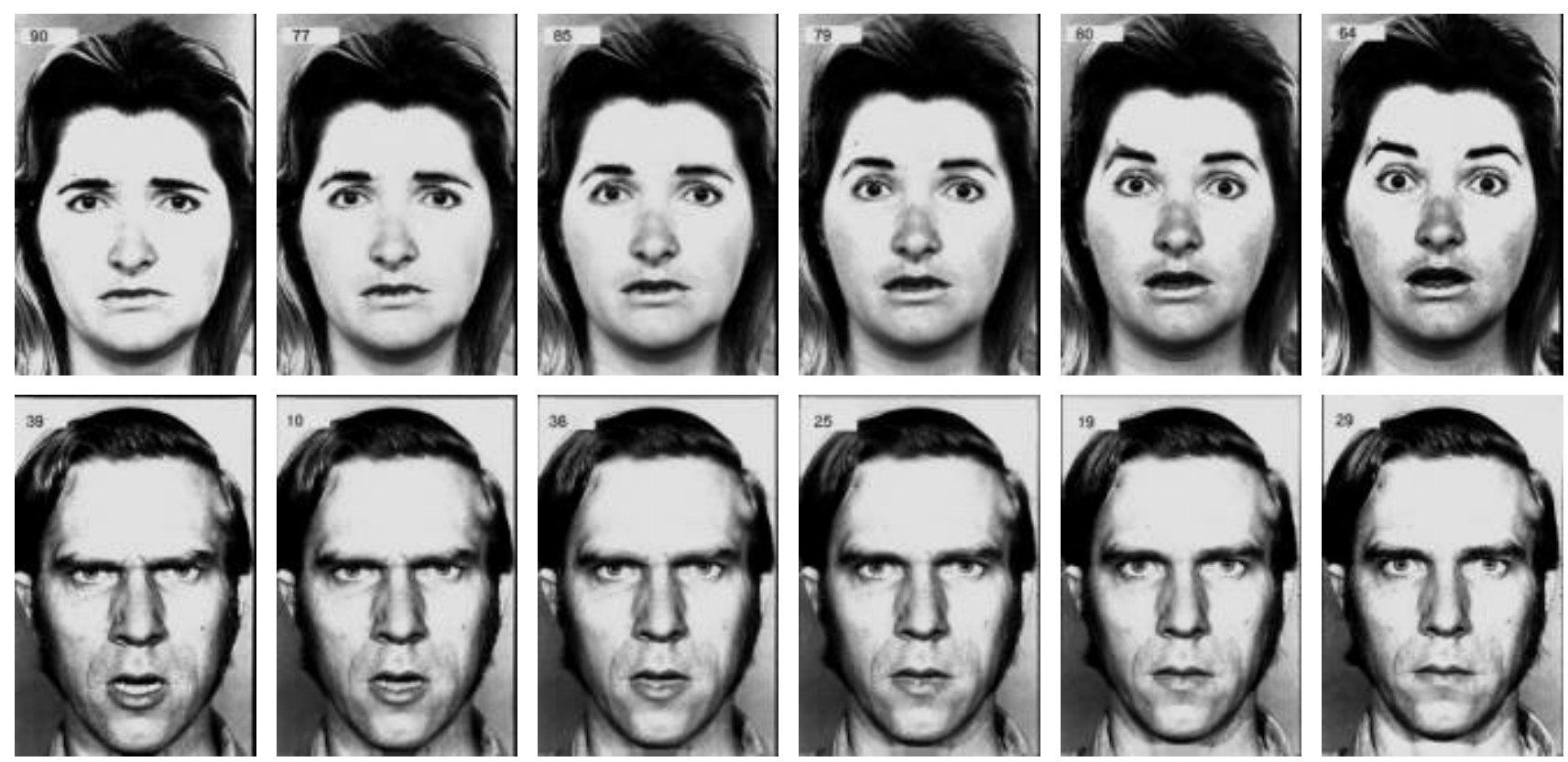

Figure 1. Examples of the morphed emotional faces: Expression continua (a) Sadness-Surprise of the MO-series, with at left 100\% Sadness, at right 100\% Surprise, and 20\%, 40\%, 60\%, 80\% morphs between them; (b) Anger-Neutral of the WF-series, with at left $100 \%$ Anger, at right $100 \%$ Neutral, and 20\%, 40\%, 60\%, $80 \%$ morphs between them.

A second series of stimuli was derived by repeating the process for another seven parent stimuli faces (e101, e103, e104, e105, e107, e108, and e110) from Ekman and Friesen (1976), all involving the male poser WF. As we are interested in those configural properties that transcend variations between posers, one consideration in the choice of these two individuals was that the expressions they use to convey particular emotions are not always identical. For instance, e61 (pure Anger, as expressed by MO) has the FACS-system description of AUs $4+5+23$, whereas e 103 (its counterpart in the WF-series) is composed of AUs $4+5+7+2+26$ (Ekman, personal communication). The latter incorporates two AUs absent in the former, "lids tight [squint]" and "jaw drop."

In addition, the parent photographs from Ekman and Friesen (1976) were enlarged and quantified using the FACEM system (Pilowsky et al., 1985), consisting of 12 measures (Figure 2). Ancillary measurements (B2 and B3) were made of the inner and outer corners of the eyebrow. To allow for direct comparisons between individual facial expressions, all measures were standardized by dividing by reference distances: that between the outer canthi of the eyes for the horizontal measures, and the length of the nose for the vertical measures. Values for the morphs were interpolated from parent-stimuli measurements. These are not true FACEM measurements, which require a final rescaling stage that is not fully documented in the available literature. Nevertheless, the codes and brief labels assigned to the measures follow the FACEM precedents.

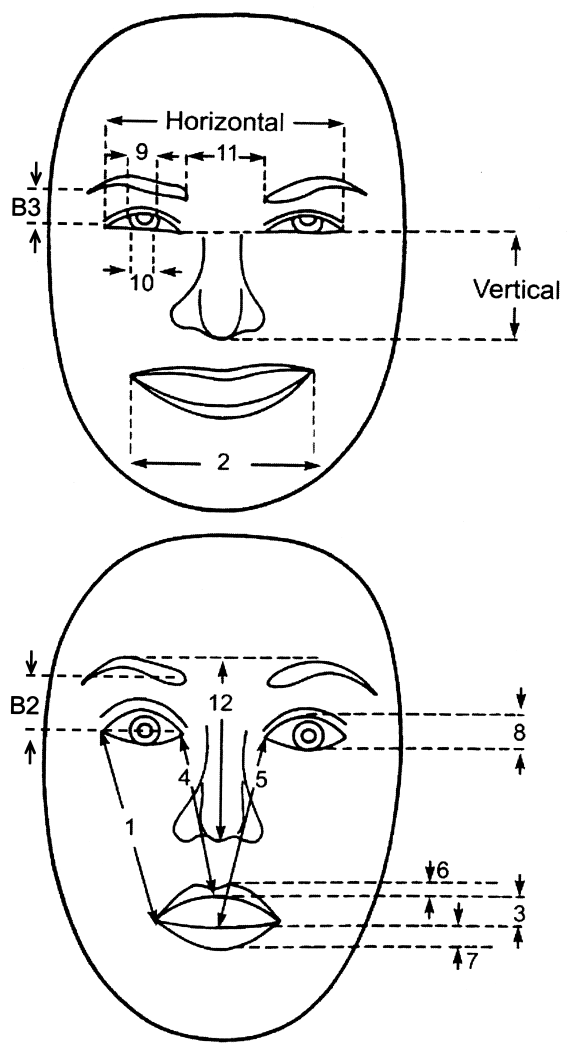

Figure 2. The objective measurements in line with the FACEM model (Pilowsky et al., 1985).

\#1 End-Lip \#6 Top-Lip Thickness \#11 Inner-Eyebrow Separation \#2 Mouth Width \#7 Lower-Lip Thickness \#12 Mid-Eyebrow \#3 Mouth Opening \#8 Eye Opening B2 Inner eyebrow \#4 Mid-Top Lip \#9 Top Eyelid / Iris Intersect B3 Outer eyebrow \#5 Mid-Lower Lip \#10 Lower Eyelid / Iris Intersect 
We normalized each measure by subtracting the value of the Neutral prototype of the MO- or WF-series (as appropriate), thus converting the absolute description of each stimulus into a description of its departure from neutrality (an option not available in most published FACEM applications). Some direct measures contain both featural and configural information: To separate these, indirect measures of the curvature and average position of lips and eyebrow were derived from direct measures, as follows:

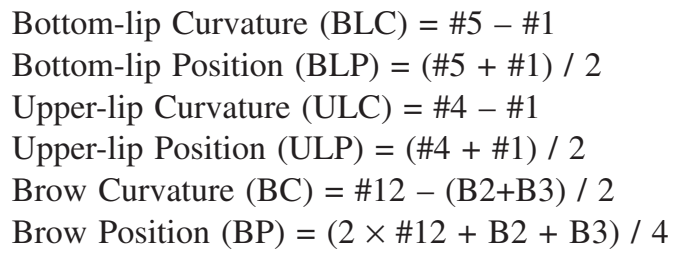

Two further measures were created from BP: Brow Raise (BR) and Brow Lower (BL). BR measures upward overall displacements of the brow from the neutral position (and is 0 otherwise) while BL measures downward displacements, together allowing for the possibility that the signal conveyed by brow displacement depends on its direction.

\section{Participants}

Informants were available from local high schools, aged between 13 and 15 years. We took this opportunity, since a comparable number of adult subjects would be hard to recruit. In the Discussion we address the question of whether data from teenagers is valid. A total of 42 students were recruited, with numbers of males and females approximately equal.

\section{Procedure}

Dissimilarity data were collected using the Method of Sorting (Emde, Kligman, Reich, \& Wade, 1978; Russell, 1980). This was elaborated into a three-phase procedure, to maximize the amount of data provided by each participant (Bimler \& Kirkland, 1997, 2001). In the first step, participants were requested to group together items which "belonged together" or were most similar. The number of groups and the number of items in each group were left up to the subjects (single-item groups were permitted). Participants were also left to make their own interpretation of "similarity." When clarification was requested, it was worded to encourage participants to reach a judgment on the basis of underlying emotion: "How similar are the emotions expressed in the photos?" or "How similar are the person's feelings?" The stimuli were described as "photographs" throughout, to disarm any suspicions as to their artificial nature.

In the second phase, participants were instructed to create and record a finer subdivision (i.e., a partition with more groups). They did this by inspecting each of the groups they had created, deciding whether the items comprising it were homogeneous in nature, and if not, how it could be split into subgroups. Finally, after restoring the original partition, participants were invited to reduce the number of groups, by selecting the two "most similar" groups and merging them into one. They repeated this merging until only two groups remained, or until the remaining groups had so little in common that nominating two of them as most similar was not possible.

Preliminary tests found that 54 stimuli were too many to be sorted at once. Accordingly, the MO- and WF-series "decks" were split into half-decks with 27 stimuli each. The splits were random (shuffling each deck first) and performed afresh for each subject. This split-deck procedure had the advantage of eliminating "anchor effects" and stereotyped patterns of sorting that might have appeared if the ends of the morphing continua had been indicated by providing the participants with all of the parent stimuli at once. There were a total of 98 sorting sequences, 49 for each of the MO- and WF-series. Each participant sorted a half-deck each of the MO- and WF-series stimuli. Fourteen participants offered to re-shuffle the items and sort a third half-deck: We decided the results were unlikely to be skewed by the greater weight of those participants in the total data pool (3\% instead of $2 \%$ ).

\section{Data Analysis}

We pooled the participants' sorting data, treating the data for the MO- and WF-series as replications. The stimuli were represented as points in a dendrogram and a multidimensional spatial model, to capture different aspects of the subjective dissimilarities among them. The dendrogram, emphasizing discontinuities and groupings among the stimuli, was obtained by applying hierarchical cluster analysis (unweighted group means algorithm) to a matrix of similarity estimates. The estimated similarity between any pair of items is simply their average "co-occurrence," that is, the proportion of partitions in which they are grouped together (e.g., Nummenmaa, 1992).

In a spatial model, the stimuli are organized into continua. A "reconstructed dyads" algorithm for multidimensional scaling (MDS) was used. Essentially, this is a way of decomposing each participant's sorting decisions into a sequence of comparisons between dissimilarities, while the points are adjusted iteratively until the geometric distances among them mirror those dissimilarities. It has been applied to images of facial affect previously (Bimler \& Kirkland, 1997, 2001).

The objective measures were used to interpret the locations of the emotional faces in this subjective representation, as affective attributes. Multiple regression analysis was applied for each measure in turn: the dependent and independent variables being respectively that measure's values for the stimuli, and the corresponding coordinates. For a given point in the MDS representation, there are two sets of FACEM measures, one from each of the MO- and WF-series. The regression was repeated separately for the two expressive gamuts as a test of generality. They were then treated as replications (like the similarity data) and analyzed in combination. 


\section{Results}

\section{Dendrogram}

Figure 3 shows the FE stimuli as nodes in a dendrogram, a non-spatial representation of subjective dissimilarities derived from the sorting data. Although the stimuli between each pair of prototype expressions differ by an equal physical amount in terms of facial-landmark displacement, the continua were subjectively discontinuous, with the stimuli arranged in seven distinct clusters: one around each prototype. Within each cluster, the corresponding prototype dominates contributions from other prototypes and obscures differences between the stimuli, a hallmark of categorical perception. The gulf separating the Happiness cluster from other expressions, corresponding to the first or leftmost branch in the dendrogram, is greatest. Next, a branch at a higher level of similarity distinguishes the Disgust cluster from the remaining "negative" expressions. The most similar cluster pairs (i.e., the last to separate, before distinctions within clusters are observed) are Sadness and Neutral, and Surprise and Fear. Paramey, Schneider, Josephs, \& Slusarek (1994) and Stringer (1967) obtained congruent results with 14 and 30 photographs respectively.

The fact that only half the prototypes (on average) were presented at one time excludes the possibility that they caused this clustering, by standing out in some way and acting as "nuclei" for the other stimuli to be grouped with. In the initial (grouping) phase, participants arranged 27 stimuli into an average of 9.5 piles. This is large enough to make it unlikely that participants were simply grouping the items into familiar expression categories.

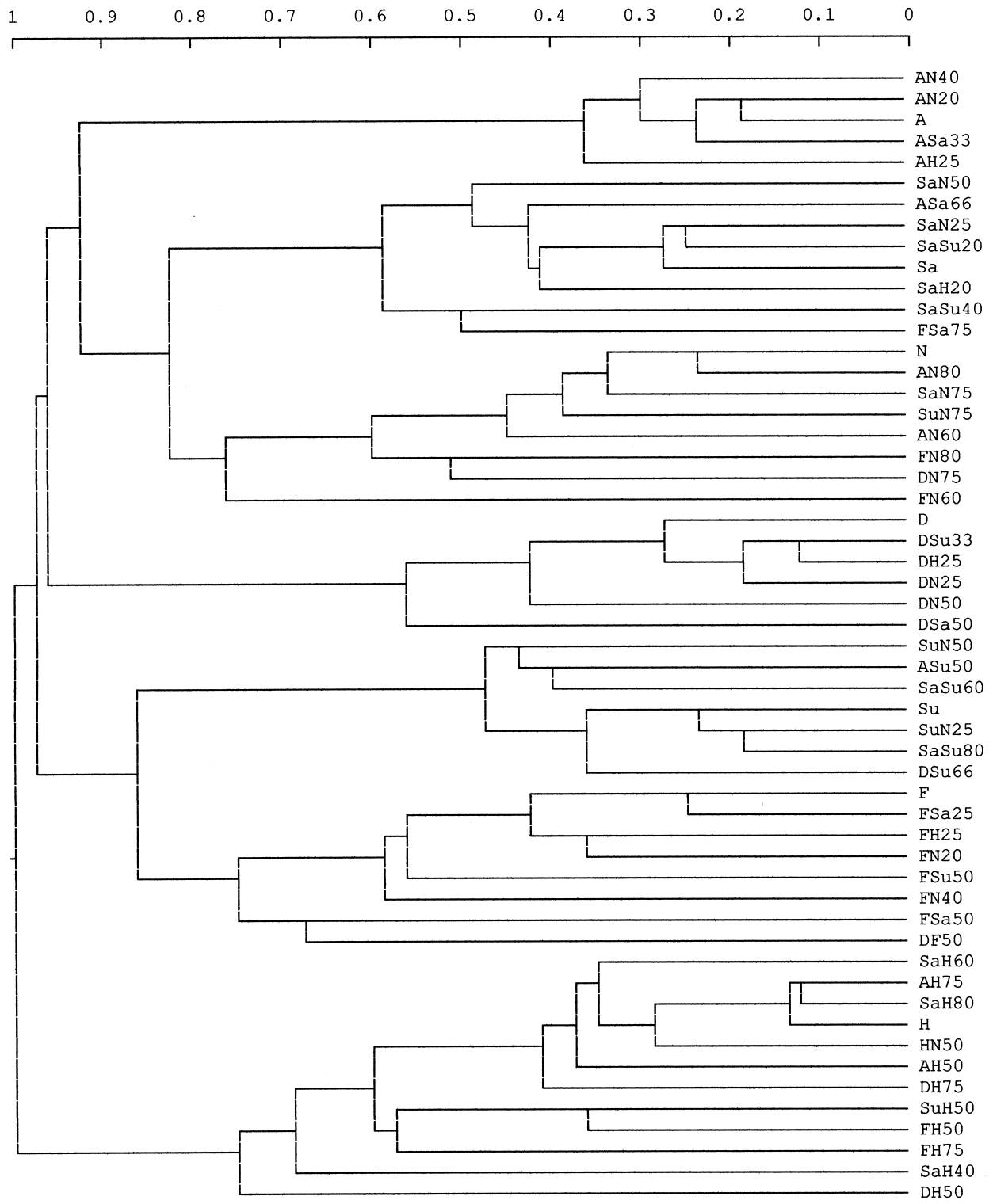

Figure 3. Dendrogram for 54 prototype and morphed facial expressions. 


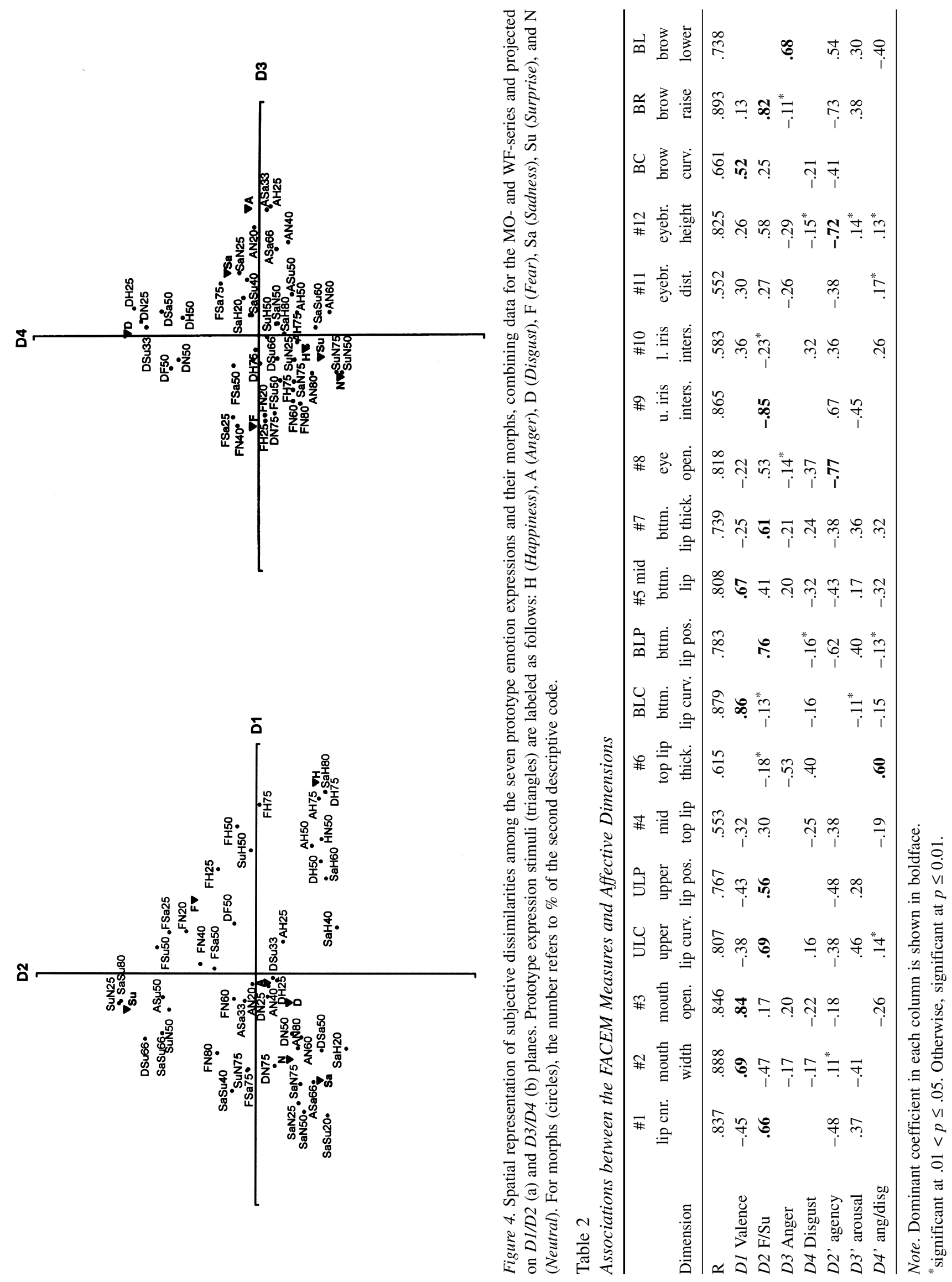




\section{Geometric Representation}

We considered MDS solutions with the number of dimensions ranging from two to five. Four dimensions seemed optimal, for reasons outlined below. The usual stress-based rules of thumb for choosing dimensionality were not applicable here since the MDS algorithm describes the goodness of fit between data and solution in terms of likelihood.

First, a maximum of four dimensions were meaningfully related to the FACEM measures, according to canonical correlation analysis (CANCORR). CANCORR compares two sets of measurements (here, the coordinates of the stimuli in "expression space" and their FACEM measures) by obtaining a linear combination of each set such that the correlation $R_{\mathrm{c}}$ between the two is maximal. The process can be repeated, extracting a second combination from each set—orthogonal to the first—with correlation $R_{2}$; and so on. Four orthogonal linear combinations of coordinates, from solutions with four or more dimensions, correlated significantly with corresponding combinations of FACEM measures. Second, the same four dimensions were present in a MDS solution for the same stimuli, obtained in using different subjects, a different procedure for eliciting dissimilarity judgments (the method of triads), and a different MDS algorithm (Kirkland, Bimler, \& Paramei, 2000). The resulting 4D solution was very similar to the present outcome: the Procrustes distance between the two was $\mathrm{g}_{l}=0.049$, while the correlation between the two matrices of reconstructed distances was $r=.85$.

The orientation of any MDS solution is arbitrary, as it can be rotated without affecting inter-point distances or goodness of fit. We rotated the 4D solution to two orientations (i.e., two sets of axes). The criteria for the first were to reflect the categorical structure of the dendrogram (in effect, emphasizing "simple structure") and to maximize the number of FACEM measures having a high correlation with one or another axis. In the second, one dimension corresponds to the familiar "Arousal" axis (ranging from neutral at one extreme to surprise, fear, anger, and disgust at the other).

The basic features of the configuration resemble those found in the previous MDS studies cited in the Introduction. $D 1$ was the expected axis of "Valence" in both alignments. In the first (Figure 4), D2, D3 and D4 were unipolar axes of Surprise-Fear, Anger and Disgust respectively. D2 might also be construed as "Attentional Activity" (Smith \& Scott, 1997). The expressions categorized as "fear" are differentiated from those of "surprise" by higher values of $D 4$ (i.e., they are closer to the pole of Disgust) and lower values of $D 3$ (farther from the Anger pole).

The second alignment is not shown for reasons of space. The alternative axes are $D 2^{\prime}, D 3^{\prime}$ and $D 4^{\prime} . D 2^{\prime}$ is the familiar personal agency (distinguishing Fear and Surprise from Anger and Disgust). D3' is an "Arousal" or "Intensity" gradient from neutral towards other expression prototypes. Finally, $D 4^{\prime}$ distinguishes Anger and Surprise on one hand from Disgust and Fear on the other.

\section{Regression and Correlation Analyses}

Multiple regression of the configural measures into the two alignments resulted in coefficients and $R$ values listed in Table 2.

The MO- and WF-series measurements were treated as replications: In the cause of brevity, the results of regressing the two expressive gamuts separately are not shown. The largest divergence from the combined results was observed for measures \#4, \#10, \#11, \#5, and BC, which all have low $R$. For instance, \#4 (upper lip thickness) was strongly associated with $D 1$ for MO's expressions, and with $D 4$ for those of WF. Other differences were subtler, and the overall patterns of these results were similar to those of the combined analysis.

Previous studies have featured the FACEM measures as independent variables in multivariate analyses, to account for the dimensional coordinates of FEs (Paramei \& Benson, 1998) or their emotion categories (Benson \& Katsikitis, 1995). For the present stimuli, lack of independence among the measures militates against that approach: For instance, Inner-Eyebrow Separation is correlated with the MidEyebrow measure $(r=.90)$.

The large number of items in our facial expression space has bestowed high levels of significance on many of the regression coefficients. Only the largest of these will be summarized below. Positive and negative coefficients are distinguished by the labels + and - . We are interested in measures that have a substantial coefficient for a given dimension.

Table 2 shows that the measures most strongly associated with D1 are \#2, \#3, \#5, BLC, and BC. Though other measures have significant $D 1$ components, these five come closest to being specific markers of the dimension. Specific markers for $D 2$ - putatively, Surprise-Fear-are measures $\# 1, \# 7, \# 8, \# 9$, \#12, ULC, ULP, BLP, and BR. Specific markers for $D 3$ are \#6 and $\mathrm{BL}$, while consistent $D 4$ markers are lacking.

The arrangement of expressions along D1, "Valence", makes it clear that this is primarily a "lower face" dimension. A high $D 1$ value is associated with measures descriptive of mouth shape, and of lip curvature and position. In addition, the lower-eyelid/iris intersect increases (\#10+), and the eyebrows are curved $(\mathrm{BC}+)$.

Beyond $D 1$, the dimensions combine aspects of both upper and lower face. High values of D2-putatively, Surprise-Fear-are characterized by widened eyes (\#8+, \#9-, \#10-), and raised eyebrows (BR+). The mouth is pursed $(\# 2-)$ with lowered corners (\#1+, BLP+) that increase upperlip curvature (ULC+) and lower-lip thickness (\#7+). For comparison, Smith and Scott (1997) link Attentional Activity with " $[\ldots]$ raised eyebrows and a variety of activities around the eyes" (p. 237).

High values of D3-Anger-are characterized by compressed lips (\#6- [and \#7-]), and lowered eyebrows (\#12-, BL+). 
High values of D4-Disgust-are characterized by a closed mouth (\#3-), with raised bottom lip (\#5-) and thick upper lip (\#6+); the eyes are narrowed (\#8-), primarily by a raised lower eyelid (\#10+).

The alternative orientation does not provide any overall gain in clarity. In particular, D3' lacks strong associations with any of the objective measures: There does not appear to be any specific measure signifying "Intensity" of facial expressions in general. Numerous measures that distinguish fear and surprise from other expressions are reinterpreted as weaker markers of personal agency, D2' (two exceptions are $\# 8$ and \#12, which are associated more compellingly with $D 2^{\prime}$ than with $D 2, D 3$ or D4 separately). Given its positive and negative associations with $D 4$ and $D 3$ respectively, it is no surprise to find that \#6 (upper lip thickness) discriminates between anger and disgust, that is, it has a large D4' coefficient in the alternative set of dimensions.

\section{Discussion}

The youthfulness of our informants might have influenced the results. This seems unlikely, however, as observers aged 13 to 15 have matured enough to perform close to adult level in tasks of facial-expression recognition (Kolb, Wilson, \& Taylor, 1992) and sorting (Bimler \& Kirkland, 2001). As mentioned above, in a replication of the MDS solution (Kirkland et al., 2000), informants were aged 18 to 21 .

In the regression analyses, we assumed that the contribution of each facial expression measure is linear and independent of context. The observed categorical-perception effect implies that this is only an approximation. Analyses which allowed for non-linear contributions or higher-order interaction between measures might result in higher correlations.

The correlates of $D 1$ found here contain no surprises. Measurements of the lower face are a recurring theme in similar studies (Pilowsky et al., 1985). In a MDS study of FACS-instructed facial expressions, Paramei and Benson (1998) labeled one of the three dimensions as "Hedonic Tone," with correlations with measures \#1, \#3, and especially \#2though not as strong as here. Those authors suggested that \#2 (mouth width) suffices to distinguish positive and negative emotions. A taxonomic clustering algorithm has been applied to FACEM measurements of 161 posed facial-expression images (Pilowsky \& Katsikitis, 1994) and 125 FACSinstructed expression images (Benson \& Katsikitis, 1995), both times finding a cluster of expressions distinguished by a similar combination of measures and identified as "Happy."

The novelty among the correlates of D2 is the weakness of the association with measure \#3. Increased \#3 was among the distinguishing features of the Surprise/Fear class of expressions described by Benson and Katsikitis (1995). At a more abstract level, facial actions related to "openness"raised eyebrows, raised upper lip, and open mouth-have been associated with a personal agency dimension (Smith
\& Scott, 1997, Table 10.2). Paramei and Benson (1998) list measures of mouth openness (\#3+, \#5+), eye openness (\#8+, $\# 9$-, \#10-), and raised eyebrow (\#12+) as the correlates of a personal agency dimension that differentiated expressions of fear and surprise from all others in a MDS treatment of FACS-instructed expressions (Paramei, 1996). For outlined emotional faces, openness of mouth and eyes was the hallmark of the most salient dimension (Paramei, 1996; Paramey et al., 1994).

We propose that mouth openness confounds separate messages sent by the curvature of the top and bottom lips, and that these are more elemental qualities (though here they are obtained indirectly from measure \#3). In Table 2, curvature was a signal of surprise or fear when manifested in the top lip, more than in the mouth overall. Bottom-lip curvature was instead associated with $D 1$ (the bottom lip's average position is however associated with $D 2$ ).

This is a convenient place to note the possible limitations of configural measures. Collectively, the FACEM scales quantify expressions of happiness well, which is not surprising, as they arose from a physical model of facial expressiveness designed specifically to synthesize smiles (Pilowsky et al., 1985). The features of surprise and fear are also quantified adequately, with several measures correlating highly and specifically with $D 2$ of the configuration. D3 and $D 4$, however, do not have such specific correlates. Applying numerical taxonomy to facial expressions, to classify them on the basis of their FACEM values, leaves room for improvement in discriminating negative emotions (Pilowsky \& Katsikitis, 1994), even when the task is simplified by omitting neutral expressions (Benson \& Katsikitis, 1995). It could be that expressions of anger and disgust are Gestalts which are not so susceptible to this kind of reductionist approach; in other words, that these emotions are conveyed by specific combinations of components, so that quantifying the parts does not capture the whole.

From the existence of the D3' axis in the alternative alignment, one might deduce that there is something in common among the "intense," "aroused" emotions at one extreme, distinguishing them from neutral at the other. However, no common quality shows up as a FACEM measure strongly associated with $D 3^{\prime}$.

It may be that expanding the suite of objective measurements would yield a higher correlation. As Kearney and McKenzie (1993) note, certain expression components cannot be measured in linear displacements, so a more sophisticated system would supplement such data by analyzing patterns of reflectance and texture. Measurements of local image features such as wrinkling, or the area of sclera visible beside the iris, were shown to provide significant information, complementary to that gained from spatial analysis (Bartlett et al., 1996). Also among the possibilities are the binary measures (the presence or absence of qualities such as forehead lines, inter-eyebrow furrows, nose wrinkles), used to good effect by Benson (1999) as supplements to the 
FACEM measures in classifying expressions into emotional categories. However, the utility of facial wrinkles is restricted by their low visibility in younger posers.

Another source of information about emotional faces is the optical flow of the transitions between facial expressions. These spatio-temporal measures have been shown to be sufficient for categorizing basic expressions (Bartlett et al., 1996; Essa \& Pentland, 1995), though considerable redundancy could be expected between these measurements of the direction and speed of optical flow and FACEM measurements of how that flow displaces facial landmarks.

\section{Conclusions}

One goal of our research was to investigate the dimensions required to accommodate FEs in a geometrical model. Previous studies have used fewer stimuli, thereby limiting the number of dimensions that they could recover with any confidence. Here, at least four dimensions seem to be necessary to match the dissimilarities among stimuli (as in Calder, Burton, Miller, Young, \& Akamatsu, 2001). The robustness of the MDS solution benefits from our use of morphing to distribute the stimuli roughly equally through expression space (in the same way that triangulating between a greater number of landmarks results in a more accurate map). In addition, morphing facilitates the measurement of the FACEM variables.

We rotated the solution to interpretable axes, scrutinizing one set of dimensions in detail, that is, one set of candidates for the affective attributes that observers use in assessing the expressions. These dimensions were chosen as ones that appeared to best reflect the categorical nature of the stimuli, as seen in a dendrogram (Figure 3). Previous studies have often retained the unrotated solution provided by the MDS software (e.g., Katsikitis, 1997), but as that default alignment is arbitrary, interpretations of its axes are problematic.

Labels were ascribed to the axes, according to the locations of the expression prototypes (Figure 4). They bear comparison with the four unipolar dimensions-Pleasure, Surprise-Fear, Anger, and Rejection (i.e., disgust)-in Nummenmaa and Kauranne (1958). Obviously, other attributes can be derived from other alignments of the MDS solution, as a rotation of the solution alters the coordinates of the stimulus points; it also alters the projections, onto the axes, of the vectors representing the FACEM measures.

We characterized this particular set of expressions by measuring facial-expression "primitives." The next step is to compare these measures to the dimensions of the geometrical "map" that represents the observed dissimilarities between the same stimuli. Underlying those dissimilarities are subjective emotional attributes, which we analyze in terms of their topographical correlates, that is, of the expressions qua spatial configurations or patterns.

The correlations between the dimensions of expression space and measurements of expression components provide some novelties, including the decoupling of top-lip and bottom-lip signals. Perhaps the key point is the fact that significant correlations exist. This was not an a priori certainty. It is not predicted by the purely categorical position (Smith \& Scott, 1997) that components lack any consistent intrinsic meanings that constrain the way in which they are assembled into expressions.

An association between mouth widening and membership of the Happiness category, for instance, or brow-lowering and Anger, would not be surprising. But here, associations were sought between the objective measures and continuous dimensions. The difference is that Valence (for instance) is an attribute of all the stimuli; a full description of FEs, even those categorized as other than "happy," requires their Valence coordinates. For a good fit between the geometrical model and measurements on a given scale, the relationship between measurements and affective attributes must hold true for all FEs, not merely those within a given category. Even happy expressions should acquire a tinge of anger from brow-lowering. This is why the regression coefficients listed in Table 2 and discussed at length are not merely paraphrasing a FACEM-measure description of the emotion prototypes. The coefficients and the values of $R$ are global properties of the MDS solution, determined by the locations in it of all the stimuli.

These results depend on the affective gamuts of two individuals, and ideally they would be replicated with other individuals. A good omen for the generality of our results is their compatibility with earlier studies using different posers and fewer stimuli (Paramei \& Benson, 1998), or following the categorization paradigm (Benson \& Katsikitis, 1995; Pilowsky \& Katsikitis, 1994). In particular, the absence of spatio-visual correlates for Intensity (Paramei \& Benson, 1998) was replicated. It may be that this attribute is best described as a facial expression's degree of departure from an implicit prototype of "neutrality," that is, as a holistic quality not easily reduced to configural relations (Calder et al., 2000a).

The difference in emotional implications of upper-lip and lower-lip curvature seems plausible, but has not been previously reported. Previous studies have subsumed these sources of configural information under "overall openness" (Paramey et al., 1994) or "lip position." This underlines the need to find the right level of measurement and suggests lines for future study.

Given the robustness of facial-expression perception, and its primacy in our social lives, one would expect it to be highly redundant, using information from a plurality of sources. We have demonstrated that facial-expression processing can make use of relative-location information. This is not to say that it uses only such information; there is ample evidence to the contrary. Katsikitis (1997, p. 620) reached a similar conclusion, that "[...] first, observers make a global assessment of a face in terms of the degree of pleasantness it shows and, second, judges rely on facial landmarks to provide clues in the delineation of one emotion from another." 
For robust transmission of affect, if a facial signal is misread due to inadequate information, it should at least be read as a similar emotion to the intended one. Thus one would expect subjectively similar emotions to be signaled by expressions that are similar in terms of objective physical appearance. Reassuringly, an application of principal components analysis to the physical appearances of a set of FEs found such a parallelism (Calder et al., 2001). Four canonical discriminant functions (dimensions) were significant, with the second and third correlating with subjective ratings of Arousal and Valence. When configural and textural aspects of each stimulus were separated, both contributed to discrimination by emotion.

We observed that in both tree and multidimensional models, morphed expressions cluster into basic-emotion categories (as in Bimler \& Kirkland, 2001). This agrees with the evidence that morphed facial expressions are recognized by their fit to discrete categories (Young et al., 1997). Yet some phenomena are hard to understand without employing dimensional concepts, such as "expression after-images," where exposure to (for instance) a happy expression makes subsequent stimuli more likely to be seen as sad (e.g., Hsu \& Young, 2004; Russell \& Fehr, 1987). Moreover, the earlier considerations of robust transmission imply that a category account is incomplete, as it has no place for the particularly high dissimilarity of happy and sad expressions, or the use of less-dissimilar and more-confusable expressions to communicate easily-exchanged emotions. An analogy is often drawn between emotional faces and colors (e.g., Calder et al., 1996), reminding us that the categorical identification of colors can coexist comfortably with a dimensional representation, and bringing to mind the arguments (Lemay et al., 1995; Smith \& Ellsworth, 1985; Smith \& Scott, 1997) that the same compatibility can apply to facial expressions.

Facial-expression processing mechanisms have been postulated that are tuned to basic-emotion configurations (Etcoff \& Magee, 1992). But paraphrasing Ortony and Turner's (1990) argument, the plasticity of such configurations allows some degree of componential variation, unless one requires them to be entirely fixed entities that cannot be decomposed into components. Components can be viewed as configural measurements of the emotional face (among other interpretations), and as such, comprise physical continua. As Beale and Keil (1995) conjecture, such lowlevel perceptual continua may constitute a basis for constructing higher-level categories or "structural invariants," consisting in the present context of discontinuous categories of FEs. Sufficiently non-linear response can produce categorical perception: "a physical continuum need not be perceptually linear" (Calder et al., 1996, p. 114). This view does not preclude a mental linear mirroring of the physical continuum of facial measurements by continuous perceptual dimensions serving as a means of affective attribution of FEs, providing consciousness with another form of access to the same information beside category membership.

\section{References}

Abelson, R.P., \& Sermat, V. (1962). Multidimensional scaling of facial expressions. Journal of Experimental Psychology, 63, 546-554.

Alvarado, N. (1996). Congruence of meaning between facial expressions of emotion and selected emotion terms. Motivation and Emotion, 20, 33-61.

Alvarado, N., \& Jameson, K. (1996). New findings on the contempt expression. Cognition and Emotion, 10, 379-407.

Bartlett, M.S., Viola, P.A., Sejnowski, T.J., Golomb, B.A., Larsen, J., Hager, J.C., \& Ekman, P. (1996). Classifying facial action. In D. Touretzky, M. Mozer, \& M. Hasselmo (Eds.), Advances in neural information processing systems, Vol. 8 (pp. 823-829). Cambridge, MA: MIT Press.

Beale, J.M., \& Keil, F.C. (1995). Categorical effects in the perception of faces. Cognition, 57, 217-239.

Benson, P.J. (1999). A means of measuring facial expressions and a method for predicting emotion categories in clinical disorders of affect. Journal of Affective Disorders, 55, 179-185.

Benson, P.J., \& Katsikitis, M. (1995). Production, reproduction, measurement and objective analyses of facial expressions. Paper presented to $3^{\text {rd }}$ Meeting of European Society for Philosophy and Psychology, Oxford, UK.

Bimler, D., \& Kirkland, J. (1997). Multidimensional scaling of hierarchical sorting data applied to facial expressions. Scandinavian Journal of Psychology, 38, 349-357.

Bimler, D., \& Kirkland, J. (2001). Categorical perception of facial expressions of emotion: Evidence from multidimensional scaling. Cognition and Emotion, 15, 633-658.

Calder, A.J., Burton, A.M., Miller, P., Young, A.W., \& Akamatsu, S. (2001). A principal components analysis of facial expressions. Vision Research, 41, 1179-1208.

Calder, A.J., Rowland,D., Young, A.W., Nimmo-Smith, I., Keane, J., \& Perrett, D.I. (2000a). Caricaturing facial expressions. Cognition, 76, 105-146.

Calder, A.J., Young, A.W., Keane, J., \& Dean, M. (2000b). Configural information in facial expression perception. Journal of Experimental Psychology: Human Perception \& Performance, 26, 527-551.

Calder, A.J., Young, A.W., Perrett, D.I., Etcoff, N.L., \& Rowland, D. (1996). Categorical perception of morphed facial expressions. Visual Cognition, 3, 81-117.

Cliff, N., \& Young, F.W. (1968). On the relation between unidimensional judgments and multidimensional scaling. Organizational Behavior and Human Performance, 3, 269-285.

De Gelder, B., Teunisse, J.-P., \& Benson, P.J. (1997). Categorical perception of facial expressions: Categories and their internal structure. Cognition and Emotion, 11, 1-23.

Ekman, P. (1993). Facial expression and emotion. American Psychologist, 48, 384-392.

Ekman, P., \& Friesen, W.V. (1976). Pictures of facial affect. Palo Alto, CA: Consulting Psychologists Press.

Ekman, P., \& Friesen, W.V. (1978). Facial Action Coding System. Palo Alto, CA: Consulting Psychologists Press. 
Emde, R.N., Kligman, D.H., Reich, J.H., \& Wade, T.D. (1978). Emotional expression in infancy: I. Initial studies of social signaling and an emergent model. In M. Lewis \& L.A. Rosenblum (Eds.), The development of affect (pp. 125-148). New York: Plenum.

Essa, I.A., \& Pentland, A. (1995). Facial expression recognition using visually extracted facial action parameters. In M. Bischel (Ed.), Proceedings of the International Workshop on Automatic Face- and Gesture-Recognition (pp. 35-40). Zurich, Switzerland.

Etcoff, N.L., \& Magee, J.J. (1992). Categorical perception of facial expressions. Cognition, 44, 227-240.

Frijda, N.H. (1969). Recognition of emotion. In L. Berkowitz (Ed.), Advances in Experimental Social Psychology, Vol. 4 (pp. 167223). New York: Academic Press.

Hsu, S.-M., \& Young, A.W. (2004). Adaptation effects in facial expression recognition. Visual Cognition, 11, 871-899.

Katsikitis, M. (1997). The classification of facial expressions of emotion: A multidimensional-scaling approach. Perception, 26, 613-626.

Katsikitis, M., Pridmore, S., \& Marzullo, M. (1999). The Facial Expression Measurement System in the assessment of the efficacy of transcranial magnetic stimulation in the treatment of depression. European Review of Applied Psychology, 49, 123-128.

Keaney, G.D., \& McKenzie, S. (1993). Machine interpretation of emotion: Design of a memory-based expert system for interpreting facial expressions in terms of signaled emotions. Cognitive Science, 17, 589-622.

Kirkland, J., Bimler, D., \& Paramei, G.V. (2000). Categorical and attributional perception of facial expressions - comparing upright and inverted faces. Perception, 29, 39S.

Kolb, B., Wilson, B., \& Taylor, L. (1992). Developmental changes in the recognition and comprehension of facial expressions: Implications for frontal lobe function. Brain and Cognition, 20, 74-84.

Lemay, G., Kirouack, G., \& Lacouture, Y. (1995). Expressions faciales émotionnelles spontanées dynamiques et statiques: comparaison d'études de jugement catégoriel et dimensionnel. Canadian Journal of Behavioural Science, 27, 125-139.

Morishima, S. (1996). Modeling of facial expression and emotion for human communication system. Displays, 17, 15-25.

Nummenmaa, T. (1992). Pure and blended emotion in the human face. Psychometric experiments. Helsinki: Suomalainen Tiedeakatemia.

Nummenmaa, T., \& Kauranne, U. (1958). Dimensions of facial expression. Report of the Department of Psychology No. 20. Institute of Pedagogics, Jyväskylä, Finnland.

Ortony, A., \& Turner, T.J. (1990). What's basic about basic emotions? Psychological Review, 97, 315-331.

Osgood, C.E. (1966). Dimensionality of the semantic space for communication via facial expressions. Scandinavian Journal of Psychology, 7, 1-30.

Paramei, G.V. (1996). [Outlined faces: Can they convey 'emotional states'?] Psikhologicheskii Zhurnal, 17(1), 70-85 (in Russian).

Paramei, G.V., \& Benson, P.J. (1998). A facial topology mediates decoding of facial expressions. Perception, 27, 132S.
Paramey, G.V., Schneider, K., Josephs, I., \& Slusarek, M. (1994). Identification of emotional meaning in line drawings of faces. Report No. 44. Ruhr-Universität, Bochum, Germany

Pilowsky, I., \& Katsikitis, M. (1994). The classification of facial emotions: A computer-based taxonomic approach. Journal of Affective Disorders, 30, 61-71.

Pilowsky, I., Thornton, M., \& Stokes, B. (1985). A microcomputerbased approach to the quantification of facial expressions. Australasian Physical and Engineering Sciences in Medicine, 8, 70-75.

Royal, D.C., \& Hays, W.L. (1959). Empirical dimensions of emotional behavior. Acta Psychologica, 15, 419.

Russell, J.A. (1980). A circumplex model of affect. Journal of Personality and Social Psychology, 39, 1161-1178.

Russell, J.A., \& Fehr, B. (1987). Relativity in the perception of emotion in facial expressions. Journal of Experimental Psychology: General, 116, 223-237.

Russell, J.A., \& Fernández-Dols, J.M. (1997). What does a facial expression mean? In J.A. Russell \& J.M. Fernández-Dols (Eds.), The psychology of facial expression (pp. 3-30). Cambridge: Cambridge University Press.

Schlosberg, H. (1954). Three dimensions of emotion. Psychological Review, 61, 81-88.

Sergent, J., Ohta, S., MacDonald, B., \& Zuck, E. (1994). Segregated processing of facial identity and emotion in the human brain: A PET study. Visual Cognition, 1, 349-369.

Smith, C.A., \& Ellsworth, P.C. (1985). Dimensions of cognitive appraisal in emotion. Journal of Personality and Social Psychology, 48, 813-838.

Smith, C.A., \& Scott, H.S. (1997). A componential approach to the meaning of facial expressions. In J.A. Russell \& J.M. Fernández-Dols (Eds.), The psychology of facial expression (pp. 229-254). Cambridge: Cambridge University Press.

Stringer, P. (1967). Cluster analysis of non-verbal judgements of facial expressions. British Journal of Mathematical and Statistical Psychology, 20, 71-79.

Takehara, T., \& Suzuki, N. (2001). Robustness of the twodimensional structure of recognition of facial expression: Evidence under different intensities of emotionality. Perceptual and Motor Skills, 93, 739-753.

Wallbott, H.G., \& Ricci-Bitti, P. (1993). Decoders' processing of emotional facial expression - a top-down or bottom-up mechanism? European Journal of Social Psychology, 23, 427-443.

Yamada, H. (1993). Visual information for categorizing facial expression of emotions. Applied Cognitive Psychology, 7, 257-270.

Young, A.W., Rowland, D., Calder, A.J., Etcoff, N.L., Seth, A., \& Perrett, D.I. (1997). Facial expression megamix: Tests of dimensional and category accounts of emotion recognition. Cognition, 63, 271-313.

Young, M., \& Yamane, S. (1992). Sparse population coding of faces in the inferotemporal cortex. Science, 256, 1327-1331.

Received November, 30, 2005 Review received, February, 13, 2006 Accepted February 20, 2006 